\title{
Direct simulation of fountains with intermediate Froude and Reynolds numbers
}

\author{
Wenxian Lin* $\quad$ S. W. Armfield ${ }^{\dagger}$
}

(Received 8 August 2003; revised 8 December 2003)

\begin{abstract}
Fountains with intermediate Froude and Reynolds numbers are of fundamental interest, especially for understanding the mechanism of turbulence and entrainment in turbulent fountains. However there has been little work done to investigate the behavior of these transitional fountains. In this work, the transient behavior of axisymmetric fountains with $1 \leq \operatorname{Fr} \leq 8$ and $200 \leq \operatorname{Re} \leq 800$ is studied by direct numerical simulation. It is found that when $R e \leq 200$, there is little mixing between the downflow of the fountain and the ambient, even for Fr as high as 8; However, when Re $\geq 400$, the mixing between
\end{abstract}

*School of Aerospace, Mechanical and Mechatronic Engineering, University of Sydney, NSW 2006, Australia; and Solar Energy Research Institute, Yunnan Normal University, Kunming 650092, CHINA. mailto:wenxian. lin@aeromech.usyd.edu. au

${ }^{\dagger}$ School of Aerospace, Mechanical and Mechatronic Engineering, University of Sydney, NSW 2006, Australia

See http://anziamj.austms.org.au/V45/CTAC2003/Lin1/home.html for this article, (C) Austral. Mathematical Soc. 2004. Published February 18, 2004. ISSN 1446-8735 
the fountain downflow and the ambient becomes stronger, indicating that the entrainment and the turbulence are mainly due to Re and the contribution from Fr is much smaller; The maximum fountain penetration height $z_{m}$ fluctuates, even when the flow reaches the quasi-steady state, due to the interaction between the downflow and the bottom boundary and/or the entrainment between the downflow and the ambient; The averaged $z_{m}$ scales with $\operatorname{FrRe}^{1 / 4}$ for $1<\operatorname{Fr} \leq 8$ and $100 \leq \operatorname{Re} \leq 800$.

\section{Contents}

1 Introduction

2 Numerical methods

3 Results

4 Conclusions

References

\section{Introduction}

Fountains are common both in industry and in nature. In any process where a dense fluid is steadily injected upward into a miscible and less dense fluid (or in the reverse case, when lighter fluid is injected downward into a denser ambient fluid) a fountain-type structure forms.

Extensive studies have been carried out on turbulent fountains with high values of $\operatorname{Fr}(\gg 1)$ and $\operatorname{Re}(\geq 800)$ (see, for example, $[1,2,3,4,5]$ ), where $\operatorname{Fr}$ 
and Re are the Froude number and Reynolds number:

$$
\mathrm{Fr}=\frac{V_{0}}{\left(R_{0} \sigma_{0}\right)^{1 / 2}}, \quad \operatorname{Re}=\frac{V_{0} R_{0}}{\nu},
$$

in which $V_{0}, R_{0}, \sigma_{0}$, and $\nu$ are, respectively the discharge velocity, source radius, reduced gravity between the fountain and the ambient fluid, and kinematic viscosity of the fluid. For these turbulent fountains, the maximum fountain penetration height $Z_{m}$ was found to scale with Fr:

$$
\frac{Z_{m}}{R_{0}}=C_{1} \mathrm{Fr},
$$

where $C_{1}$ is a proportional constant found experimentally to be in the range of 2.06 to $2.88[1,2,4,5]$.

For fountains with a weak source, the influence of the discharge momentum of the fountain is equal to or less than that of the negative buoyancy and $Z_{m}$ is of the order of $R_{0}$. The fountain is then characterized by small values of $\operatorname{Fr}(\leq 1.0)$ and $\operatorname{Re}(\leq 500)$. Such fountains have no distinguishable upward and downward flows, instead the streamlines curve and spread from the source. Our recent analytical and numerical studies showed that these weak fountains demonstrate different behavior patterns to those of turbulent fountains $[6,7,8,9,10]$. It was found that $Z_{m}$ has the following scaling relation with Fr and Re,

$$
\frac{Z_{m}}{R_{0}}=C_{2} \operatorname{FrRe}^{-1 / 2}
$$

where $C_{2}$ is another proportional constant. The behavior of unsteady fountains with intermediate values of $\operatorname{Fr}(1 \leq \mathrm{Fr} \leq 20)$ and $\operatorname{Re}(200 \leq \operatorname{Re} \leq 1000)$, which are in the transitional regime from laminar to turbulent flow, is of fundamental interest for understanding the generation mechanism of turbulence and entrainment within turbulent fountains. Despite this no studies have been found in which the behavior of these fountains is investigated, which motivated us to carry out the current study. 
In this study, we investigate the behavior of unsteady axisymmetric fountains with the intermediate values of $1 \leq \mathrm{Fr} \leq 8$ and $200 \leq \mathrm{Re} \leq 800$ by direct numerical simulation. The major findings are that the entrainment and the turbulence are mainly due to Re and the contribution from Fr is much smaller, the maximum fountain penetration height fluctuates, even when the flow reaches the quasi-steady state, due to the interaction between the downflow and the bottom boundary and/or the entrainment between the downflow and the ambient, and the averaged maximum fountain penetration height scales with $\operatorname{FrRe}^{1 / 4}$ for $1<\mathrm{Fr} \leq 8$ and $100 \leq \operatorname{Re} \leq 800$.

\section{Numerical methods}

The physical system under consideration is a vertical circular container containing a Newtonian fluid initially at rest and at temperature $T_{a}$, the sidewall is non-slip and insulated and the top is open. On the bottom centre, an orifice with radius $R_{0}$ is used as the fountain discharge source. The remaining bottom region is a rigid non-slip insulated boundary. At time $t=0$, a stream of fluid at $T=T_{0}<T_{a}$ is injected impulsively into the container from the source to initiate the fountain and this discharge is maintained thereafter. Due to the symmetry of the system geometry and the boundary and initial conditions as well as the weak fountain discharge, the fountain flow is axisymmetric and laminar.

The flow is described by the following Navier-Stokes and temperature equations, which are written in non-dimensional form in cylindrical coordi- 
nates with the Boussinesq assumption as,

$$
\begin{aligned}
& \frac{1}{r} \frac{\partial}{\partial r}(r u)+\frac{\partial v}{\partial z}=0 \\
& \frac{\partial u}{\partial \tau}+\frac{1}{r} \frac{\partial}{\partial r}(r u u)+\frac{\partial}{\partial z}(v u)=-\frac{\partial p}{\partial r}+\frac{1}{\operatorname{Re}}\left\{\frac{\partial}{\partial r}\left[\frac{1}{r} \frac{\partial}{\partial r}(r u)\right]+\frac{\partial^{2} u}{\partial z^{2}}\right\} \\
& \frac{\partial v}{\partial \tau}+\frac{1}{r} \frac{\partial}{\partial r}(r u v)+\frac{\partial}{\partial z}(v v)=-\frac{\partial p}{\partial z}+\frac{1}{\operatorname{Re}}\left[\frac{1}{r} \frac{\partial}{\partial r}\left(r \frac{\partial v}{\partial r}\right)+\frac{\partial^{2} v}{\partial z^{2}}\right]+\frac{1}{\mathrm{Fr}^{2}} \theta(6) \\
& \frac{\partial \theta}{\partial \tau}+\frac{1}{r} \frac{\partial}{\partial r}(r u \theta)+\frac{\partial}{\partial z}(v \theta)=\frac{1}{\operatorname{RePr}}\left[\frac{1}{r} \frac{\partial}{\partial r}\left(r \frac{\partial \theta}{\partial r}\right)+\frac{\partial^{2} \theta}{\partial z^{2}}\right]
\end{aligned}
$$

where $\operatorname{Pr}$ is the Prandtl number. In these equations all lengths, velocities, time, pressure, and temperatures are made dimensionless by $R_{0}, V_{0}, R_{0} / V_{0}$, $\rho V_{0}^{2},\left(T_{0}-T_{a}\right)$, respectively, where $\rho$ is the fluid density.

The direct numerical simulations have been carried out using a finite volume method. The governing equations are discretized on a non-staggered mesh, with standard second-order central differences used for the viscous, pressure gradient, and divergence terms, whereas the QUICK third-order upwind scheme is used for the advective terms. The momentum and temperature equations are solved using an ADI scheme. The second-order AdamsBashforth scheme and Crank-Nicolson scheme are used for the time integration of the advective terms and the diffusive terms, respectively. To enforce the continuity, the pressure correction method is used to construct a Poisson equation, which is solved using the preconditioned generalized minimum residual (GMRES) method. A nonuniform mesh of $298 \times 297$ grids has been used in this study to enable the regions of rapid solution variation to be accurately resolved while limiting the total number of nodes. Detailed descriptions of these schemes were given in $[6,7]$ and the code has been successfully used for the simulation of a range of buoyancy dominated flows. 


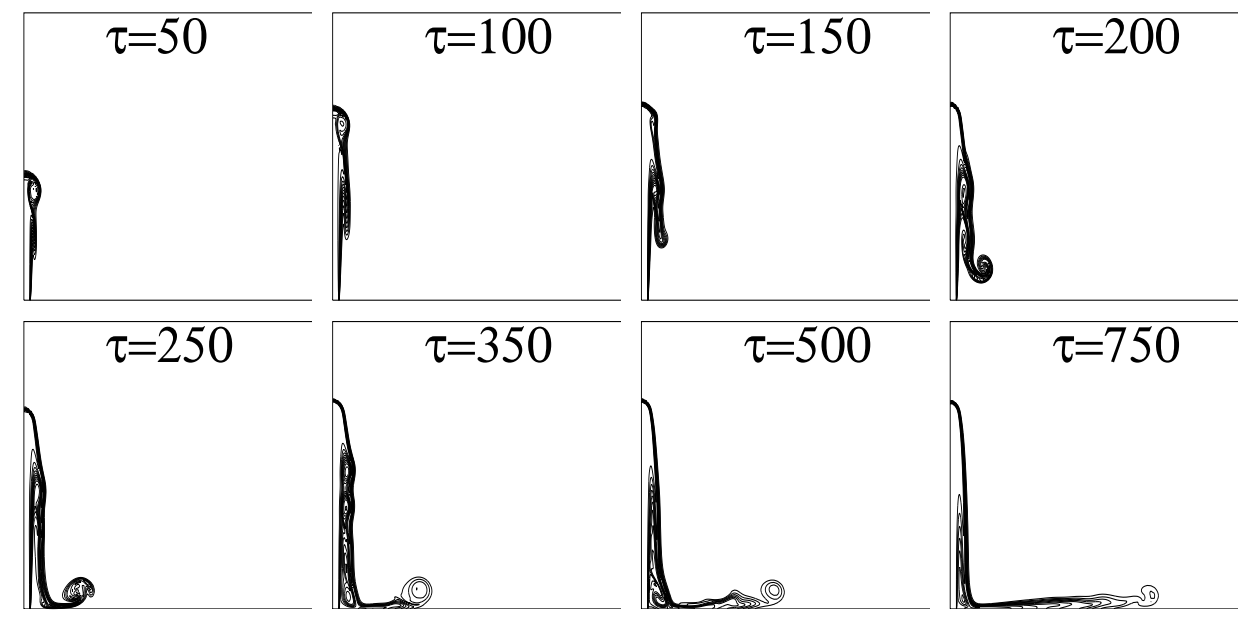

Figure 1: The temperature contours at times $\tau=50,100,150,200,250$, 350,500 , and 750 for $\mathrm{Fr}=8$ and $\mathrm{Re}=200$.

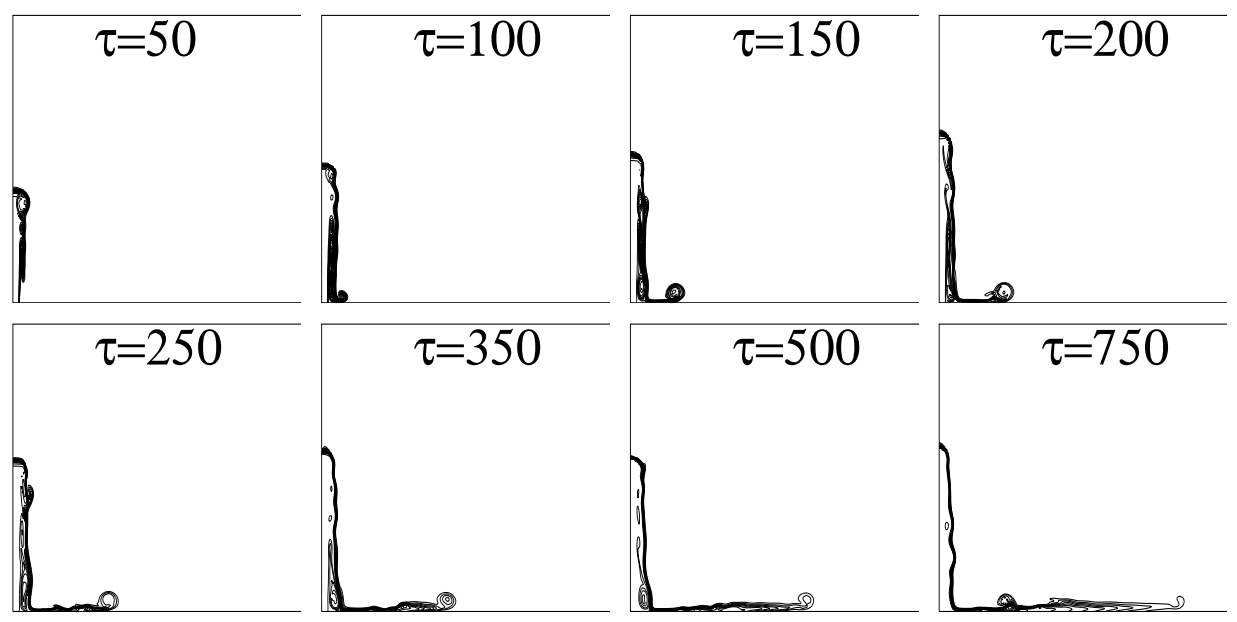

Figure 2: The temperature contours at times $\tau=50,100,150,200,250$, 350,500 , and 750 for $\mathrm{Fr}=6$ and $\mathrm{Re}=400$. 

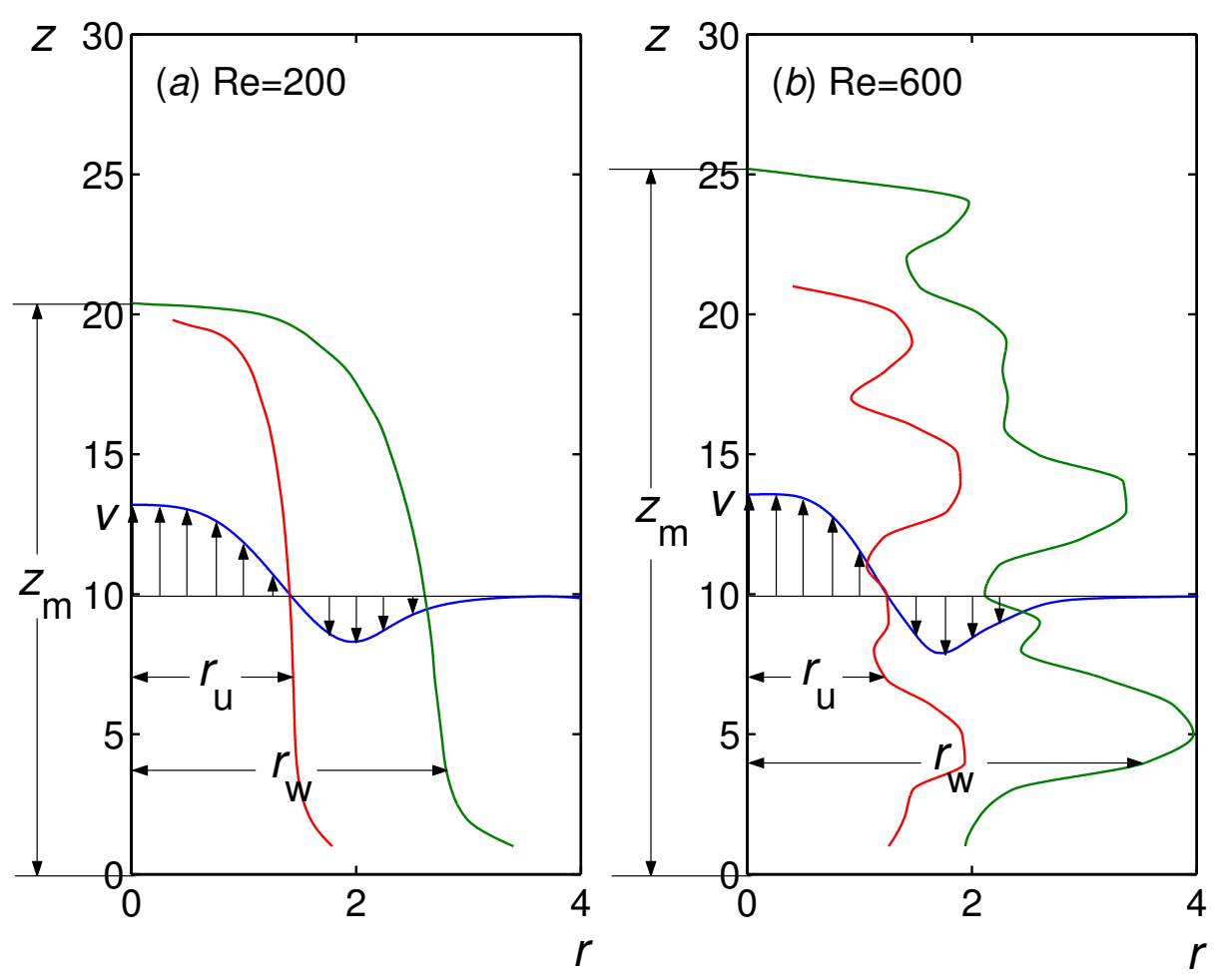

Figure 3: The profiles of the fountain width $r_{w}$ based on the temperature $\theta=-0.01$ contour and the upflow width $r_{u}$ based on the vertical velocity $v=0$ contour for: (a) $\operatorname{Re}=200$; and (b) $\operatorname{Re}=600$ with $\operatorname{Fr}=6$ at $\tau=750$. 

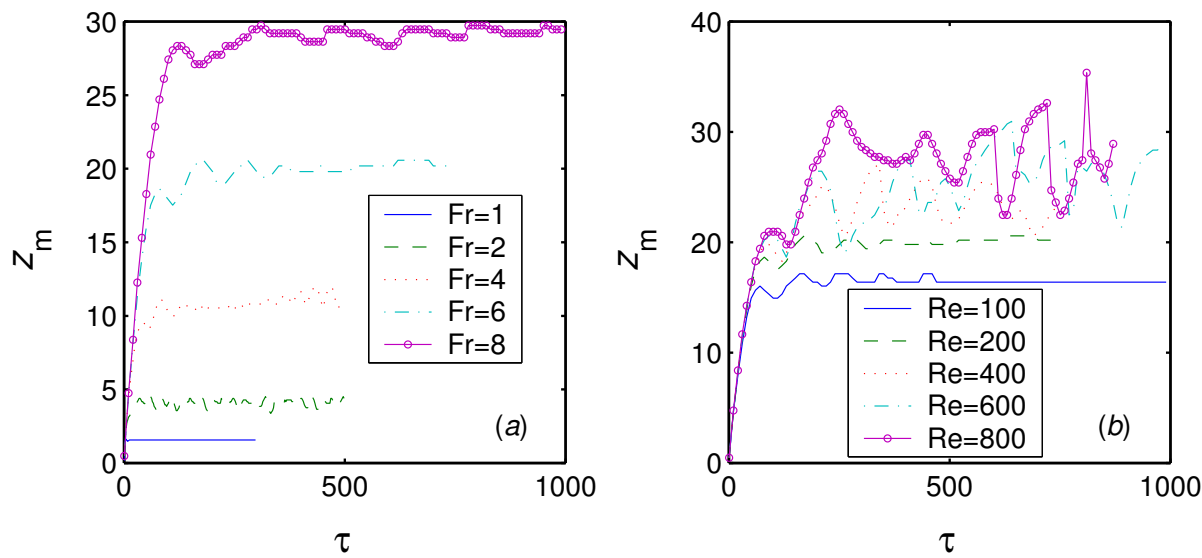

Figure 4: The time series of $z_{m}$ for: (a) $\mathrm{Fr}=1,2,4,6$ and 8 with $\operatorname{Re}=200$; and (b) $\operatorname{Re}=100,200,400,600$ and 800 with $\mathrm{Fr}=6$.

\section{$3 \quad$ Results}

To show the behavior of fountains with intermediate values of Fr and Re, a series of direct numerical simulations have been carried out for axisymmetric fountains with selected values of Fr and Re. Specifically, $\mathrm{Fr}=1,2,4,6$ and 8 with $\operatorname{Re}=200$ and $\operatorname{Pr}=7$ are used to show the dependence on Fr, and $\operatorname{Re}=100,200,400,600$ and 800 with $\mathrm{Fr}=6$ and $\operatorname{Pr}=7$ are used to show the dependence on Re.

To provide an overview of the transient behavior of fountains with intermediate values of Fr and Re, visualizations of the numerically simulated transient temperature contours at 8 specific moments are presented in Figure 1 for $\mathrm{Fr}=8$ and $\mathrm{Re}=200$ and Figure 2 for $\mathrm{Fr}=6$ and $\mathrm{Re}=400$. The major flow patterns observed are that when Re $\leq 200$, there is little mixing between the downflow of the fountain and the ambient, even for $\mathrm{Fr}$ as high as 8 . However, when $R e \geq 400$, the mixing between the downflow of the fountain and the ambient becomes stronger, indicating that the entrain- 

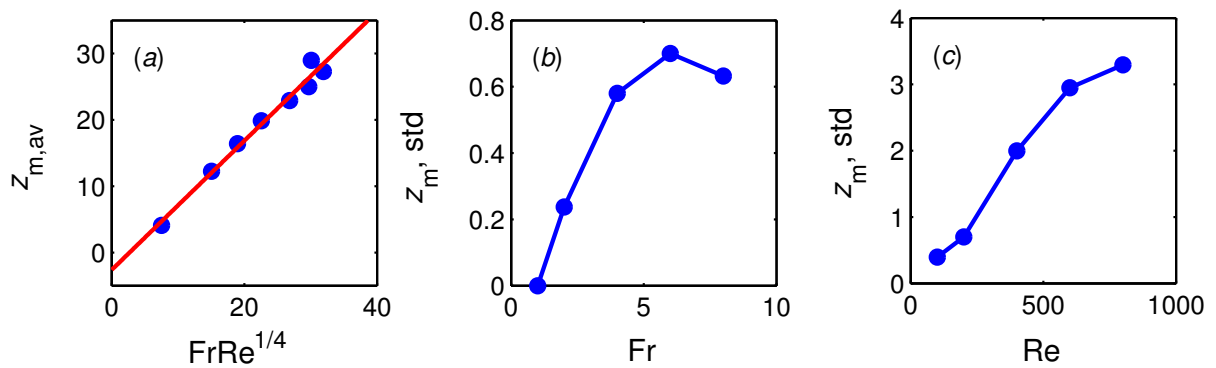

FiguRE 5: (a) $z_{m, a v}$ against $\operatorname{FrRe}^{1 / 4}$; and standard deviation of $z_{m, a v}$ against Fr, (b), and Re, (c), for $2 \leq \mathrm{Fr} \leq 8$ and $100 \leq \operatorname{Re} \leq 800$.
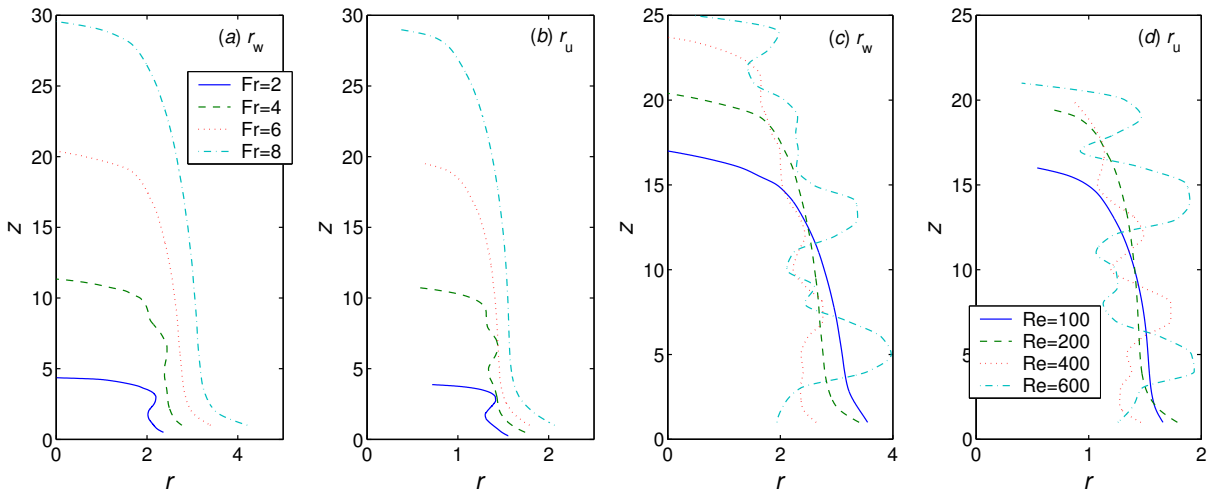

Figure 6: The profiles of the fountain width $r_{w}$ and the upflow width $r_{u}$ with selected values of Fr and Re. 
ment and further the turbulence are mainly due to Re and the contribution from Fr is much smaller, as clearly shown in Figure 3. It is also found that the maximum fountain penetration height $z_{m}$, where $z_{m}$ is non-dimensionalized by $R_{0}$, fluctuates instead of keeping constant, even when the flow reaches the quasi-steady state, as it should do when $\mathrm{Fr} \geq 1$ [6].

The magnitudes and frequencies of the fluctuations of $z_{m}$ for selected values of $\mathrm{Fr}$ and Re are presented in Figure 4. The fluctuations are the results of the interaction between the fountain downflow and the bottom boundary and/or the mixing (entrainment) between the fountain downflow and the ambient.

For fountains with intermediate values of $\mathrm{Fr}$ and Re, it is expected that $z_{m}$ has the following scaling relation with Fr and Re,

$$
z_{m} \sim \operatorname{Fr} f(\operatorname{Re}),
$$

where ' $\sim$ ' denotes 'scales with' and $f(\mathrm{Re})$ represents a 'function of Re'. The specific form of the function can be determined experimentally and/or numerically.

The numerical results presented in Figure 5(a) give the following scaling relation for $1<\operatorname{Fr} \leq 8$ and $100 \leq \operatorname{Re} \leq 800$,

$$
z_{m, a v}=0.974 \operatorname{FrRe}^{1 / 4}-2.623 .
$$

where $z_{m, a v}$ is the averaged maximum fountain penetration. The standard deviations of $z_{m, a v}$, which represent the magnitudes of the fluctuations of $z_{m}$, that is, the interactions between the fountain downflow and the bottom boundary and/or the entrainment between the fountain downflow and the ambient, are presented in Figure 5(b) and 5(c) for different values of Fr and Re, respectively. These results and the numerical results presented in Figure 6, where the profiles of the fountain width $r_{w}$ and the upflow width $r_{u}$ are shown for selected values of Fr and Re, give further confirmation that the entrainment and the turbulence are mainly due to Re and the contribution from Fr is much smaller. 


\section{Conclusions}

1. When $\operatorname{Re} \leq 200$, there is little mixing between the downflow of the fountain and the ambient, even for Fr as high as 8. However, when Re $\geq 400$, the mixing between the downflow of the fountain and the ambient becomes stronger, indicating that the entrainment and further the turbulence are mainly due to Re and the contribution from Fr is much smaller.

2. The maximum fountain penetration height $z_{m}$ fluctuates instead of staying constant, even when the flow reaches the quasi-steady state, resulting from the interaction between the fountain downflow and the bottom boundary and/or the mixing (entrainment) between the fountain downflow and the ambient.

3. Numerical results show that the averaged maximum fountain penetration height $z_{m, a v}$ scales to $\mathrm{Fr}$ and $\mathrm{Re}^{1 / 4}$ for $1<\mathrm{Fr} \leq 8$ and $100 \leq \operatorname{Re} \leq 800$.

Acknowledgements: The financial support of the Australian Research Council, via an Australian Postdoctoral Fellowship (DP0449876), from the National Natural Science Foundation of China (10262003), and the Natural Science Foundation of Yunnan Province of China (Key Project 2003E0004Z), are gratefully acknowledged.

\section{References}

[1] B. R. Morton. Forced plumes. J. Fluid Mech., 5:151-163, 1959. C67, C68 
[2] J. S. Turner. Jets and plumes with negative or reversing buoyancy. J. Fluid Mech., 26:779-792, 1966. C67, C68

[3] E. J. List. Turbulent jets and plumes. Ann. Rev. Fluid Mech., 14:189-212, 1982. C67

[4] T. Mizushina, et al.. An experimental study of vertical turbulent jet with negative buoyancy. Wärme- und Stoffübertragung, 16:15-21, 1982. C67, C68

[5] W. D. Baines, J. S. Turner and I. H. Campbell. Turbulent fountains in an open chamber. J. Fluid Mech., 212: 557-592, 1990. C67, C68

[6] W. Lin and S. W. Armfield. Direct simulation of weak axisymmetric fountains in a homogeneous fluid. J. Fluid Mech., 403:67-88, 2000. C68, C70, C75

[7] W. Lin. Scaling analysis and direct simulation of unsteady weak fountain and natural convection flow. Ph. D thesis, The university of Sydney, Australia, 2000. C68, C70

[8] W. Lin and S. W. Armfield. Direct simulation of weak laminar plane fountains in a homogeneous fluid. Int. J. Heat Mass Transfer, 43:3013-3026, 2000. C68

[9] W. Lin and S. W. Armfield. Weak fountains in a stratified fluid. Phys. Rev. E, 66:066308, 2002. C68

[10] W. Lin and S. W. Armfield. The Reynolds and Prandtl number dependence of weak fountains. Computational Mechanics, 31:379-389, 2003. C68 